\title{
Aux origines du monachisme en Gaule : Les communautés martiniennes du IVe siècle
}

The Beginnings of Monasticism in Gaul: Martinian Communities in 4th Century

\section{Lucien-Jean Bord}

\section{(2) OpenEdition}

\section{Journals}

\section{Édition électronique}

URL : http://journals.openedition.org/abpo/2467

DOI : $10.4000 /$ abpo. 2467

ISBN : 978-2-7535-2136-0

ISSN : $2108-6443$

Éditeur

Presses universitaires de Rennes

Édition imprimée

Date de publication : 30 octobre 2012

Pagination : 13-26

ISBN : 978-2-7535-2134-6

ISSN : 0399-0826

\section{Référence électronique}

Lucien-Jean Bord, «Aux origines du monachisme en Gaule : Les communautés martiniennes du IVe siècle », Annales de Bretagne et des Pays de l'Ouest [En ligne], 119-3 | 2012, mis en ligne le 30 octobre 2014, consulté le 01 mai 2019. URL : http://journals.openedition.org/abpo/2467 ; DOI : 10.4000/ abpo. 2467 


\title{
Aux origines du monachisme en Gaule : les communautés martiniennes du IV siècle
}

\author{
Fr. Lucien-Jean BORD, o.s.b. \\ Abbaye Saint-Martin F-86240 Ligugé
}

\section{La fondation ligugéenne}

" Hilaire étant déjà passé, il suivit ses pas jusqu'à Poitiers. Ayant reçu de lui le plus gracieux accueil, il s'installe un ermitage non loin de la ville ${ }^{1}$.»

Ces deux lignes de Sulpice Sévère constituent non seulement la première mention de la présence de Martin en Poitou ${ }^{2}$ mais aussi l'attestation de la fondation d'un " ermitage ". Un siècle plus tard, dans sa vie de saint $M a r t i n^{3}$, rédigée entre 458 et $488^{4}$, Paulin de Périgueux écrit :

1. "Cum iam Hilarius praeterisset, Pictauos eum est uestigiis persecutus; cumque ab eo gratissime fuisset exceptus, haut longe sibi ab oppido monasterium conlocauit ", (Vita 7, 1; I, p. 266-267). Les citations de la Vita, ainsi que les mentions de cet ouvrage que nous serons amené à faire dans la suite de cette étude renvoient toutes à l'édition de Jacques Fontaine (SulPICE SÉvÈre, Vie de saint Martin, Sources Chrétiennes 133-135, éd. FonTAINE, Jacques, Paris, 1967-1969); par souci de commodité, nous l'indiquerons par le seul mot Vita suivi des mentions de texte et de pagination.

2. Rappelons que la Vita Martini de Sulpice Sévère, grâce à une lettre de Paulin de Nole à l'auteur, peut être datée de 397 Or, 397, c'est l'année même de la mort de Martin, décédé à Candes (cf. SulPICE SÉvère, Vita, III, p. 1285, n. 2), ce qui fait de Sulpice Sévère, qui avait été " moine " martinien, un témoin direct. Paulin de Nole écrivait à son ami Sulpice Sévère : "Tu es donc béni du Seigneur pour avoir relaté avec autant de dignité dans le style que de justesse dans les sentiments, l'histoire d'un si grand évêque et d'un confesseur aussi éclatant " (" benedictus igitur tu homo Domino, qui tanti sacerdotis et manifestissimi confessoris historiam tam digno sermone quam iusto affectu percensuisti "); cf. Paulin de Nole, Epistulae, (cSel XXIX), éd. De Hartel, Guilelmus, et Kamptner, Margit, Vienne, 1999, ep. XI, 11, p. 70. Sur la datation des lettres de Paulin de Nole, on se reportera à : Brochet, J., La Correspondance de saint Paulin de Nole et de Sulpice Sévère, Paris, 1906.

3. PAUlin de PÉRIGUeUX, De uita sancti martini episcopi libri VI, CSEL, XVI, 1, éd. PETSCHENIG, M., Vienne, 1888, p. 17-159.

4. Sur la date de la Vita de Paulin de Périgueux, cf. LABARre, Sylvie, Le Manteau partagé. Deux métaphores poétiques de la Vie de saint Martin chez Paulin de Périgueux (Ve siècle) 
"Cependant Martin apprend qu'un ordre du roi rappelle enfin de son injuste exil le saint évêque, compagnon de son illustre vie aux jours heureux où, docteur au pays des Pictaves, il enseignait à ces peuples indomptés les préceptes du salut. Ravi de joie, il se met en route avec empressement; son ardente et pieuse affection double sa vitesse. Mais Hilaire, impatient, n'avait pu l'attendre; il avait déjà quitté les remparts élevés de la superbe Rome pour se rendre au plus tôt sous les murs de Poitiers. Animé d'un saint zèle, Martin vole sur ses traces et trouve enfin l'objet de ses désirs et le terme de ses fatigues. Il construit près de cette ville un monastère, seulement pour donner du repos et du soulagement à ses membres, car son âme, pleine de Dieu, habitait les célestes demeures ${ }^{5}$."

Nous n'entrerons pas ici dans les discussions et controverses concernant et la date et le lieu de l'installation de Martin non loin de la ville de Poitiers ${ }^{6}$ - d'autant plus que la localisation Locoteiacum/Ligugé est maintenant généralement admise - notre propos étant plus de tenter de cerner quelles pouvaient être la conception et les usages du monachisme martinien de la part de son " fondateur". Relevons cependant dans la phrase de Sulpice Sévère la précision quant au lieu choisi pour l'ermitage « non loin de la ville ", ce haut longe qui est déjà un renseignement en lui-même; en effet, dans le latin classique, l'expression désigne plus une petite distance qu'une proximité immédiate ${ }^{7}$. Notons cela dès maintenant car, pour le monastère épiscopal de Marmoutier, nous rencontrons également une fondation établie à petite distance de la ville.

Malgré la pauvreté des sources concernant non seulement le monastère poitevin primitif mais aussi le mode de vie qui y était pratiqué, nous trouvons, toujours dans Sulpice Sévère, une indication précieuse sur l'objet même de son installation. Pour qualifier la " fondation " martinienne

et Venance Fortunat (VI siècle), (Collection des Études Augustiniennes, Série Antiquités, 158), Paris, 1998, p. 21-28.

5. «Interea iniusto tandem remeare refertur/sanctus as exilio per Regis iussa sacerdos/ cum quo praeclarae fuerant consortia uitae/dum Pictauorum doctor floreret in oris/indomitis tradens populis praecepta salutis/et quamquam propere sanctum rapuisset ouantem/uelox affectu pietas, tamen ille moratum/inpatiens celsae iam liquerat ardua Romae/moenia, Pictonicas citius reuehendus ad oras./Ergo legens praegressa pio uestigia cursu/promeruit uotum cordis finemque laboris/construit hic cellam fessis solacia membris/nam mens plena deo caelesti in sede mandebat " (PAulin de Périgueux, De uita, op. cit., I, 285-297, p. 30-31).

6. Pour ne parler que de la controverse très polémique du premier quart du $\mathrm{Xx}^{\mathrm{e}}$ siècle, citons : Babut, Ernest-Charles, Saint-Martin de Tours, Paris, 1912; Delehaye, Hippolyte, "Saint Martin et Sulpice Sévère ", Analecta Bollandiana 38, 1920, p. 5-136; Juluan, Camille, "La jeunesse de saint Martin " (= Note gallo-romaine 47), Revue des Études Anciennes 12, 1910, p. 260-280; ID., "Remarques critiques sur les sources de la vie de saint Martin " (= Notes gallo-romaines 93-96), Revue des Études Anciennes 24, 1922, p. $37 s q, 123 s q$ et 229 sq; ID., "Remarques critiques sur la vie et l'œuvre de saint Martin " (= Notes galloromaines 97-99), Revue des Études Anciennes 24, 1922, p. 306 sq et 25; 1923, p. 49 sq, 139 sq. et $234 s q$. Sur cette question, on se reportera en dernier lieu à BORD, Lucien-Jean, Histoire de l'abbaye Saint-Martin de Ligugé, 361-2001, Paris, 2005, p. 31-42.

7. "Non longe a domo esse " (CICÉRON, In Verram actio, 7, 94); " non longe ex eo loco abesse " (CÉSAR, De bello Gallico, V, 21, 2); « non longius milia passum octo abesse " (ibid. $\mathrm{V}, 53,7)$. 
en Poitou, Sulpice Sévère écrit, rappelons-le : haut longe sibi ab oppido monasterium conlocauit. Le verbe collocare signifie plus qu'une simple installation d'un particulier en un lieu qu'il aurait choisi; il marque dans la langue classique l'installation de colons ou la mise en position de troupes ${ }^{8}$. Ainsi que l'a bien vu Jacques Fontaine : " coloniser spirituellement des campagnes dans lesquelles règne encore le paganisme, lutter en miles Christi contre l'erreur et le mal sous toutes leurs formes, mais d'une manière plus concrète, plus sociale en quelque sorte, que ne le faisaient la plupart des solitaires d'Égypte ${ }^{9}$ ". On doit comprendre la venue de Martin à Ligugé comme un " envoi ", dans le sens missionnaire du terme, de la part de l'évêque de Poitiers, et se pose alors la question du choix de ce lieu.

Ce n'était pas un endroit trop plaisant, ni un lieu fameux, ni vraiment une thébaïde ${ }^{10}$. Par contre, il y a plus que de fortes présomptions pour y situer un lieu de culte païen fort ancien et de quelque importance ${ }^{11}$. Et l'on connaît la pugnacité avec laquelle Martin a détruit les sanctuaires païens ruraux pour en extirper le paganisme ${ }^{12}$. Qu'il nous soit donc permis de proposer une hypothèse sur le but de l'envoi de Martin dans le vallon de Locoteiacum : il se serait agi, de la part de l'évêque de Poitiers, de christianiser un lieu de culte païen non seulement en provoquant la disparition de ces monuments mais en y installant une présence " monastique ", ou plutôt anachorétique. Voilà qui éclaire d'un jour particulier la manière " active " dont le fondateur concevait l'idiorythmie anachorétique de son monastère.

Car il s'agit bien plus d'une vie anachorétique " collective " que d'un cénobitisme tel qu'il sera compris par les rédacteurs des règles monastiques ultérieures, en Occident en tout cas. La vie monastique menée à Marmoutier, peu après l'élection épiscopale de Martin au siège de Tours ${ }^{13}$, nous fournit à ce propos bien plus de renseignements. Relevons que, à Marmoutier tout comme à Ligugé, même si à l'origine Martin cherchait une

8. "Certis locis cum ferro homines conlocati »(CICÉRON, Pro A. Caecina oratio, 41); " exercitum in provinciam quae proxuma est Nimidice hiemandi gratia conlocat " (SALLUSTE, De bello Jugurthino, 61, 2).

9. Vita II, p. 612.

10. BorD, Lucien-Jean, Histoire de l'abbaye Saint-Martin de Ligugé, op. cit., p. 11-13.

11. Ibidem, p. 20-30.

12. Ibid., p. 36. Martin n'a d'ailleurs pas le privilège de ce type d'évangélisation un peu rude. Rappelons que les lois promulguées par Arcadius et Honorius, moins d'un demi-siècle plus tard, et qui ne firent que reprendre et confirmer les usages antérieurs, ordonnaient la fermeture et la destruction des temples, particulièrement des sanctuaires ruraux, la confiscation des statues, l'affectation des bâtiments ou de leurs matériaux à un usage profane; $c$. Codex Theodosianus. Theodosiani libri XVI cum Constitutionibus Sirmondianis et leges novellae ad Theodosianum pertinentes, éd. Mommsen, Theodor et MEYER, Paul, Berlin, 1904-1905, XVI, 10, 16, T. II, p. 902.

13. L'élection épiscopale de Martin et sa consécration comme évêque de Tours intervinrent pendant le règne de Valentinien I ${ }^{\text {er }}$ (364-375); la critique récente s'accorde pour situer cet événement entre 370 et 372. Cf. STANCLIFFe, Clare, St Martin and his Hagiographer. History and Miracles in Sulpicius Severus, Oxford, 1983, p. 111-133, sp. 116. 
vie solitaire, il semble bien qu'il ait été presque immédiatement rejoint par des disciples ${ }^{14}$.

\section{Les informations concernant Marmoutier}

Un monasterium, donc, avec une vie orientée vers un apostolat de conversion des païens habitant les campagnes environnantes. Si Sulpice Sévère reste très discret sur la fondation ligugéenne, il est bien plus prolixe sur Marmoutier, vraisemblablement parce qu'il y a vécu ${ }^{15}$. Il convient de scruter les propos de Sulpice Sévère sur Marmoutier :

"Pendant quelque temps, il habita donc une cellule attenante à l'église. Puis ne pouvant plus supporter d'être dérangé par ceux qui lui rendaient visite, il s'installa un ermitage à deux milles environs hors les murs de la cité. Cette retraite était si écartée qu'elle n'avait rien à envier à la solitude d'un désert. D'un côté, en effet, elle était entourée par la falaise à pic d'un mont élevé, et le reste du terrain était enfermé dans un léger méandre du fleuve de Loire; il n'y avait qu'une seule voie d'accès, et encore fort étroite. Martin occupait une cellule construite en bois, et un grand nombre de frères étaient logés de la même manière. Mais la plupart s'étaient fait des abris en les creusant dans la roche du mont qui les dominait. Il y avait environ quatrevingts disciples, qui se formaient à l'exemple de leur bienheureux maître. Là, personne ne possédait rien en propre, tout était mis en commun; Il était interdit d'acheter ou de vendre quoi que ce fut, comme bien des moines en ont l'habitude. On n'y exerçait aucun art, à l'exception du travail des copistes; encore n'y affectait-on que les plus jeunes : leurs aînés vaquaient à la prière. On ne sortait que rarement de sa cellule, sauf pour se réunir au lieu de la prière. Passée l'heure du jeûne, ils prenaient tous ensemble leur nourriture; personne ne connaissait le vin, sauf celui que la maladie y contraignait. Bon nombre s'habillaient de poil de chameau; on y tenait pour une faute grave une tenue trop raffinée. Cela doit être tenu pour d'autant plus extraordinaire qu'il y avait parmi eux, disait-on, un grand nombre de nobles; ayant reçu une éducation toute différente, ils s'étaient volontairement pliés à cette vie d'humilité et de mortification. Nous en avons vu plusieurs devenir ensuite évêques ${ }^{16}$."

14. OudART, Hervé, "Marmoutier au IV siècle, un exemple de vie érémitique? ", Revue Mabillon 65, 1993, p. 109-132, sp. p. 115-116.

15. Vita I, p. 135-139.

16. "Aliquandiu ergo adhaerenti ad ecclesiam cellula usus est; dein, cum inquietudinem se frequentantium ferre non posset, duobus fere extra ciuitatem milibus monasterium sibi statuit. Qui locus tam secretus et remotus erat, ut eremi solitudinem non desideraret. Ex uno enim latere praecisa Liger fluuius reducto paululum sinu clauserat; una tantum eademque arta admodum uia aditi poterat. Ipse ex lignis contextam celulam habebat, multique ex fratribus in eudem modum; plerique saxo superiecti montis cauato receptacula sibi fecerant. Discipuli fere octoginta erant, qui ad exemplum beati magistri instituebantur. Nemo ibi quicquam proprium habebat, omnia in medium conferebantur. Non emere aut uendere, ut plerique monachis moris est, quicquam licebat; ars operi minor aetas deputabatur : maiores orationi uacabant. Rarus cuiquam extra cellulam suam egressus, nisi cum ad locum orationis conueniebant. Cibum una omnes post horam ieiunii accipiebant. Vinum nemo nouerat, nisi quem infirmitas coegisset. Plerique camelorum saetis uestibantur: mollor ibi habitus pro crimine erat. Quod eo magis sit mirum necesse est, quod multi inter eos nobiles habebantur, 
Il convient bien entendu de faire la part des conventions de style et du renvoi obligé aux moines des déserts d'Égypte ${ }^{17}$. Nous pouvons cependant gager, sans grand risque d'erreur, que Martin transposa sur les rives de la Loire ce qu'il avait connu sur celles du Clain; d'ailleurs, Sulpice Sévère le sous-entend dans le court prologue qu'il écrit juste avant sa description de Marmoutier et où nous pouvons lire :

« En effet, avec une fermeté parfaite, il restait semblable à celui qu'il avait été auparavant. Même humilité en son cœur, même pauvreté dans son vêtement. C'est ainsi qu'il remplissait les fonctions épiscopales, plein d'autorité et de prestige, sans déserter pour autant sa profession ni ses vertus monastiques $^{18}$."

Ainsi que l'écrit Hervé Oudart : «Il apparaît que l'accession à la dignité épiscopale n'altère en rien le mode de vie de Martin, que ce soit au plus profond de lui-même, "en son cœur", ou en son "dessein monastique", ou que ce soit même dans les manifestations concrètes de son humilité ${ }^{19}$." L'expression choisie par Sulpice Sévère pour qualifier la nouvelle installation martinienne est d'ailleurs très proche de celle qu'il a utilisée pour Ligugé : sibi [...] monasterium conlocauit pour l'établissement poitevin et sibi monasterium statuit pour Marmoutier, indiquant la continuité de sa vie monastique ${ }^{20}$.

Le type d'habitat qu'il choisit de se bâtir, une cellule construite en bois, retient notre attention. Le fait tranche avec la description des lieux qui précède et qui, elle, emprunte nettement à la littérature latine classique : ainsi que l'a bien vu Jacques Fontaine ${ }^{21}$, c'est plus un paysage de l'Énéide que Sulpice Sévère déroule sous nos yeux que le rivage de la Loire bordé par une falaise point trop élevée. Seule cette lignis contextam cellulam n'appartient pas au modèle virgilien; le lyrisme de la phrase latine présente d'ailleurs une rupture comme si l'on changeait de registre. La cabane en bois ne trouve pas de parenté directe chez les ermites du désert ${ }^{22}$ et semble

qui longe aliter educati ad hanc se humilitatem et patientiam coegerant; pluresque ex eis postea episcopos uidimus. " (Vita 10, 3-8; I, p. 274-275).

17. D’emblée, Sulpice Sévère place la fondation de Marmoutier dans le cadre d'une recherche de solitude et de calme qui assimile Martin à Antoine et à Hilarion (Vita II, p. 665-666). Sur les influences orientales chez Sulpice Sévère, on pourra consulter : Gribomont, Jean, "L'influence du monachisme oriental sur Sulpice Sévère ", dans Saint Martin et son temps. Mémorial du XVte centenaire des débuts du monachisme en Gaule, 3611961, (Studia Anselmiana, 46), Rome, 1961, p. 135-149.

18. "Idem enim constantissime perseuerat qui prius fuerat. Eadem in corde eius humilitas, eadem in uestitu eius utilitas; atque ita, plenus auctoritatis et gratiae, inplebat episcopi dignitatem, ut non tamen propositum monachi uirtutemque deseret. " (Vita 10, 1-2; I, p. 272-274).

19. OudarT, Hervé, "Marmoutier au Iv ${ }^{\mathrm{e}}$ siècle ", art. cit., 65, p. 113.

20. La différence réside uniquement dans le choix du verbe : collocare à Ligugé où, nous l'avons vu, Martin est " envoyé ", statuere à Marmoutier où, évêque du lieu, il s'établit de lui-même.

21. Vita II, p. 667-672.

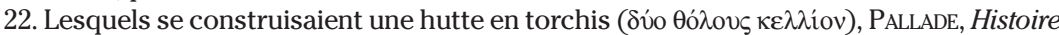
lausiaque, 8, 5, (Scrittori Greci e Latini), éd. BARTELINK, G.J.M., Milan, 1974, p. 44-45), ou 
bien renvoyer à ce que l'auteur de la Vita a pu voir par lui-même et qui correspond d'ailleurs à l'habitation du petit peuple des campagnes galloromaines, resté fidèle à la " cabane " gauloise ${ }^{23}$. Si l'on pose que Martin avait conservé ses habitudes précédentes - et tout nous pousse à le croire - il n'est pas étonnant que l'on n'ait rien retrouvé du " monastère " martinien de Ligugé qui devait, lui aussi, se composer de bâtiments de bois et de branchages.

La seconde indication concerne le nombre des disciples. Le nombre de quatre-vingts disciples ne doit pas être pris au pied de la lettre; non seulement il se rencontre à plusieurs reprises dans la Bible vétérotestamentaire $^{24}$, mais il renvoie surtout au symbolisme de l'ogdoade et à celui des "décuries " chères aux moines égyptiens ${ }^{25}$. Plus important est le qualificatif de maître qui est ici donné à Martin. C'est la seule fois que Sulpice Sévère désigne ainsi son héros et c'est justement dans le contexte de son magistère monastique, ce qui nous fournit une précieuse indication sur l'organisation de la communauté martinienne, d'autant plus que la formation dispensée aux disciples est caractérisée par le verbe instituere qui comporte une nette connotation de discipline ${ }^{26}$. Notons encore que le verbe est ici employé au passif et sans complément d'agent, ce qui peut suggérer une formation individuelle des disciples, chacun réglant sa conduite sur l'exemple du " maître ${ }^{27}$ ". Ce type de rapport entre maître et disciples n'est pas sans rappeler ce qui se pratiquait à la même époque dans les communautés égyptiennes ${ }^{28}$.

Vient ensuite une notation sur la pauvreté personnelle et la mise en commun des biens, qui se réclame évidemment de la communauté chrétienne primitive de Jérusalem (Ac 2,44), mais la formulation nous interdit de prendre ces deux phrases pour une simple note conventionnelle, obligatoire dans toute description de communauté monastique. Le langage revêt ici un caractère juridique précis avec une mise en indivis des biens (omnia in medium conferebantur) et une interdiction formelle du ius commercii; il s'agit bien de la description par un juriste - ce qu'était Sulpice Sévère ${ }^{29}$ des usages réels de la communauté martinienne. Et l'auteur va plus loin en rapportant également l'interdiction de tout travail manuel sauf une excep-

encore s'appropriaient divers lieux comme Antoine qui avait habité tour à tour un tombeau, un fort abandonné et une caverne (ATHANASE D'AlEXANDRIE, Vie d'Antoine (Sources Chrétiennes, 400), éd. BARTElinK, G.J.M., Paris, 1994, 8, 1-2, p. 156-157; 12, 3, p. 168-169; $50,1 s q$, p. 268-271).

23. GRENIER, Albert, Les Gaulois, (Petite bibliothèque Payot, 157), Paris, 1970, p. 233.

24. 2 Ch. 26, 17 ; Jer 41, 5.

25. DANiÉLou, Jean, Bible et liturgie. La théologie biblique des sacrements et des fêtes d'après les Pères de l'Église, Paris, 1951, p. 355-387; - Vita II, p. 674-675 et n. 3.

26. Philosophorum praeceptis instituta via (CICÉRON, De finibus, 4, 17).

27. OudART, Hervé, "Marmoutier au Ive siècle ", art. cit., p. 119.

28. Regnault, Lucien, La Vie quotidienne des Pères du désert en Égypte au Ive siècle, Paris, 1990, p. 145-147; DEsPREZ, Vincent, Le Monachisme primitif. Des origines jusqu'au concile d'Ephèse, (Spiritualité orientale, 72), Bégrolles-en-Mauges, 1998, p. 290-292.

29. Vita I, p. 54. 
tion sur laquelle nous reviendrons. Voici qui est " nouveau " car aussi bien les "Règles ${ }^{30}$ " que les usages rapportés par les vies et les apophtegmes des Pères du désert nous montrent anachorètes et cénobites travaillant de leurs mains pour assurer leur subsistance.

Cette interdiction de tout " art " vient immédiatement après celle de tout commerce et doit lui être rattachée. Le mot ars désigne ici très nettement les activités manuelles tendant à produire des biens manufacturés ${ }^{31}$, cela ressort de l'exception faite pour le travail des copistes. Réduire cependant la pensée de Sulpice Sévère à la simple constatation d'une prohibition serait lui faire injustice; chez lui, le mot ars sert également à désigner les artifices du diable ${ }^{32}$ et l'on ne peut écarter l'hypothèse que son insistance sur le " non labeur " des disciples de Martin ait pour but d'opposer la grandeur de la seule prière aux artifices de l'activité diabolique. Cela ne doit cependant pas nous inciter à considérer cette notation comme historiquement douteuse; on doit poser que les moines martiniens ne pratiquaient aucune activité vénale ou lucrative, se consacrant essentiellement à la prière.

Il convient alors de se demander de quoi vivaient les frères? Une fois encore, c'est le texte de Sulpice Sévère qui nous fournit une indication. Bien que la mention faite de la mise en commun des biens renvoie d'évidence à Ac 2, 44, elle ne reprend pas l'expression même des Actes des Apôtres (et habebant omnia communia) mais utilise une formule différente : omnia in medium conferebantur ${ }^{33}$. Ce medium a particulièrement retenu l'attention d'Hervé Oudart ${ }^{34}$ qui montre combien le choix de ce vocabulaire est lié à la préoccupation juridique de l'auteur. Si le sens le plus obvie de medium est " milieu, centre", dans ce cas particulier il revêt le sens de " accessible à tous ${ }^{35}$ " et l'on doit comprendre qu'il ne s'agit pas d'une mise en commun dans le sens de la communauté hiérosolymite où s'exerçait le contrôle des Apôtres, mais d'une mise à disposition de chacun sur laquelle Martin n'a pas plus de droits que les frères. Ce point, qui aurait pu paraître secondaire, nous renseigne bien plus que de longues descriptions sur la manière dont Martin concevait l'esprit devant régner dans sa communauté.

Un épisode des Dialogues, rapportant un miracle de Martin en faveur de la familia de Lycontius, doit également être pris en compte; le bénéficiaire du miracle veut prouver sa reconnaissance à l'évêque :

" Il lui apporta aussi cent livres d'argent que le bienheureux homme ne rejeta ni ne reçut, mais avant que cette libéralité ait atteint la limite du

30. Nous pensons particulièrement à celles de Pacôme, de Basile et d'Augustin.

31. Ars est donc pris dans le sens que lui donne Cicéron (De officiis, 1, 150) quand il écrit : " opifices omnes in sordida arte uersantur ".

32. "Frequenter autem diabolus, dum mille nocendi artibus sanctum uirum conabatur inludere »(Vita 22, 1; I, p. 300).

33. Vita 10, 6 (I, p. 274).

34. OUdART, Hervé, " Marmoutier au Ive siècle ", art. cit., p. 122.

35. "In medio omnibus palma est posita " (TÉRENCE, Phormio 16); « bona interfectorum in medium cedant " (TACITE, Historice 4, 64). 
monastère, il l'affecta incontinent au rachat de captifs; et comme les frères lui suggéraient d'en réserver une partie pour les dépenses du monastère, que le vivre était maigre pour tous et que beaucoup manquaient de vêtements, il dit :"l’Église nous nourrira et nous vêtira pourvu que nous fassions montre de ne rien avoir demandé pour nos besoins ${ }^{36}$." "

Notons que Martin s'arrange pour disposer de la somme, en faveur de captifs $^{37}$, avant qu'elle ne soit parvenue au monastère, sinon elle aurait relevé des dispositions relatives au medium dont nous venons de parler; mais il ne fait pas cela avec un bien lui appartenant puisque, selon le texte même, nec respuit nec recepit. Les demandes des frères, elles, nous apprennent que la communauté martinienne vivait dans une précarité certaine que semble d'ailleurs encourager son fondateur. Quant à la réponse de Martin, il ne paraît pas qu'il faille y voir une sorte d'obligation systématique pour l'Église : tout au plus devait-elle venir en aide aux frères dans les moments les plus difficiles mais à condition toutefois qu'ils se fient à la providence divine et ne demandent ni ne récriminent ${ }^{38}$. Il y a là une exigence de pauvreté et de renoncement poussée très loin.

Venons-en maintenant à la seule activité qui soit tolérée : la copie des manuscrits. Là aussi, le texte de la Vita nous apporte de précieuses indications. De toute évidence il ne s'agit pas d'un travail lucratif ni d'une activité destinée à procurer à la communauté les textes dont elle aurait eu besoin mais de l'un des éléments de la formation des jeunes moines ${ }^{39}$. En effet, nous lisons dans la même phrase que seuls les plus jeunes y étaient affectés tandis que les aînés vaquaient à la prière. Il y a un lien évident entre les deux activités qui paraissent comme les deux faces d'une même réalité : le dialogue monastique avec Dieu. Dès lors, les qualificatifs de "minor aetas "

36. "Centum etiam argenti libras obtulit quas uir beatus nec respuit nec recepit, sed priusquam pondus illud monasterii limen adtingeret, redimendis id captiuis continuo deputauit; et cum ei suggeretur a fratribus ut aliquid ex eo in sumptum monasterii reseruaret, omnibus in angusto esse uictum, multis deesse uestitum : nos, inquit, ecclesia et pascat et uestiat, dum nihil nostris usibus quaesisse uideamur. " (SULPICE SÉvĖRE, Libri qui supersunt, (CSEL, 01), éd. HaLM, C., Vienne, 1876, Dialogus II (III), 14, p. 212).

37. Le rachat des captifs, otages enlevés par les barbares ou soldats prisonniers, était une œuvre d'Église recommandée par AmBroISE DE MiLAN (Expositio Psalmi CXVIII, 8, 41, (CSEL, 62), éd. Petschenig, Michael, et Zelzer, Michaela, Vienne, 1999, p. 176) et qui fera peu après l'objet d'une constitution d'Honorius la reconnaissant comme l'une des charges officielles de l'Église (Codex Theodosianus IX, 3, 7; op. cit., t. II, p. 442-443); cf. PIÉTRI, Luce, La ville de Tours du IV au vie siècle : naissance d'une cité chrétienne, (Collection de l'École Française de Rome, 68), Rome, 1983, p. 695.

38. La question d'une aide régulière ou ponctuelle reste néanmoins ouverte car nous ne disposons pas d'élément déterminant permettant de trancher dans un sens ou dans l'autre. Cf. Vita II, p. 677, n. 2; OuRY, Guy-Marie, Saint Martin de Tours, l'homme au manteau partagé, Chambray-lès-Tours, 1987, p. 97; OudART, Hervé, " Marmoutier au Iv siècle ", art. cit., p. 128 et n. 63.

39. Jacques Fontaine (Vita II, p. 677) écrit à ce propos : " Mais comment penser que cette main-d'œuvre composée de mineurs ait été capable de "produire" - et d'écouler une quantité de manuscrits suffisante pour entretenir 80 personnes, même soumises au régime ascétique le plus strict? " 
et de " maiores " ne doivent plus être compris dans un sens juridique ou civil mais bien comme qualifiant ceux qui sont plus ou moins avancés dans la vie monastique ${ }^{40}$.

La suite du récit nous fournit quelques indications sur le quotidien de la vie des frères - garder la cellule, jeûner et s'assembler pour la prière - et sur les us vestimentaires - tunique de poils, absence de raffinement - qui renvoient non seulement à Jean le Baptiste et aux Pères du désert mais cadrent bien avec ce que nous savons du monachisme gaulois au IV siècle $^{41}$. Par contre, la sortie contre les tenues trop raffinées, même si elle évoque une réalité vestimentaire des moines, semble bien appartenir dans sa forme au fond littéraire de Sulpice Sévère qui emprunte son molliores habitus à Stace $^{42}$.

Enfin, l'indication sur la composition sociale de la communauté martinienne est particulièrement intéressante puisque nous apprenons qu'un nombre non négligeable de "frères " étaient issus de la noblesse galloromaine. Ici encore, le fait est corroboré par ce que nous savons de l'engouement des jeunes nobles pour un genre de vie évangélique, entraînant d'ailleurs les critiques et les récriminations de la haute société qui considérait cette fuite du monde comme un véritable " abandon de poste ${ }^{43}$ ". Jacques Fontaine ${ }^{44}$ relève avec pertinence que Sulpice Sévère profite de cette mention des nobiles pour opposer un argument aux détracteurs du monachisme naissant : la communauté de Marmoutier fonctionnait comme une véritable pépinière d'évêques !

Le portrait qui nous est ainsi tracé du " monastère " de Marmoutier s'inscrit fort bien dans ce que nous savons par ailleurs des premières communautés monastiques chrétiennes en Gaule. On ne peut certes prendre le mot monasterium dans l'acception qu'il acquerra plus tard; s'il ne s'agit déjà plus de ces monastères familiaux ${ }^{45}$ qui ont fleuri en Italie, nous ne

40. À titre de comparaison, on peut se reporter à une correspondance de saint Jérôme (c. 347-420) où l'on peut lire : "j'ai des élèves qui s'adonnent à l'art du copiste " (habeo alumnos qui antiquariae arti seruiant); JÉRÔME, Lettres. Tome I, (Collection des Universités de France), éd. LaBourT, Jérôme, Paris, 1949, ep. V, 2, p. 19.

41. Il semble bien que les tuniques en poil de chameau ne soient pas une concession stylistique de Sulpice Sévère à la tradition du monachisme oriental et égyptien mais aient constitué la " marque " des ascètes gallo-romains du IV siècle. Ces objets étaient vraisemblablement rapportés par de pieux voyageurs si l'on en croit Paulin de Nole qui remercie son ami Sulpice Sévère de lui avoir fait parvenir des "pallium tissés en poil de chameau " (pallia camelorum pilis texta); PAULIN DE NOLE, Epistulae, op. cit., ep. XXIX, 1, p. 247. Cf. également Vita II, p. 681-682.

42. "Neque enim ille dedisset, si molles habitus et tegmina foeda fateri ausa seni "; STACE, Achilléide, (Collection des Universités de France), éd. MÉHEust, Jean, Paris, 1971, I, 142, p. 12.

43. Maraval, Pierre, Le Christianisme de Constantin à la conquête arabe, (Nouvelle Clio), Paris, 1997, p. 280-281.

44. Vita II, p. 683.

45. Ce type de monachisme a été particulièrement bien étudié par PETERSEN-SzEMERÉDY, Griet, Zwischen Weltstadt und Wüste : Römische Asketinnen in der Spätantike, Göttingen, 1993. 
sommes pas encore en présence des monastères organisés qui vont peupler progressivement tout l'Occident à partir du ve siècle. Le monachisme martinien tel qu'il nous est décrit par Sulpice Sévère - en faisant bien entendu la part d'une certaine exagération littéraire et apologétique de la part de l'auteur ${ }^{46}$ - appartient à un type intermédiaire qui organisait des communautés de semi-ermites sans pour autant recourir à une règle établie, codifiée et acceptée.

Quelle image pouvons-nous donc nous faire de cette communauté? Une fraternité anachorétique d'ascètes issus pour une bonne part de la noblesse gallo-romaine, vivant le renoncement et la pauvreté en un même lieu, mais chacun dans son habitation, ayant un certain nombre d'activités communes, jeûnant, se consacrant essentiellement à la prière, à la méditation et à la lectio. Les plus " jeunes " dans la "vie monastique " copiaient les textes, plus semble-t-il par souci de formation que dans un but de diffusion des écrits. L'environnement était solitaire, retiré, pauvre et on ne procédait à aucune construction en matériaux durables, se contentant d'aménager des grottes ou d'élever des cabanes de bois et de branchages. Le fait que l'on y refusait toute activité vénale nous renseigne sur la spiritualité des ascètes qui voulaient se consacrer uniquement et entièrement à la recherche de Dieu.

Peut-on exporter rétroactivement ce modèle sur Ligugé? Si cela est quelque part hypothétique, force nous est d'admettre que Martin n'a pas inventé du jour au lendemain son monachisme en arrivant à Tours, qu'une communauté s'était déjà groupée autour de lui sur les rives du Clain et que le type de vie qu'on y menait a été repris, au moins en grande partie, lors de la fondation de Marmoutier. Nous avons d'ailleurs vu que Sulpice Sévère suggérait une telle continuité. Nous pensons que l'ensemble des points que nous venons de voir a pu s'appliquer à l'établissement martinien de Ligugé, y compris pour l'origine sociale des disciples ou le rejet du travail vénal.

Ainsi que nous l'avons déjà dit, si on ne peut pas encore parler de " monastère " dans le sens classique du terme, on ne peut non plus parler " d'ermitage ». Le monasterium de Sulpice Sévère dit bien ce que son auteur veut nous signifier.

\section{Les origines du monachisme martinien}

Reste à nous interroger sur les origines du monachisme martinien; en d'autres termes, quelles influences ont pu déterminer les choix du fondateur de Ligugé et de Marmoutier?

Il semble bien que des traditions orales concernant Antoine l'aient atteint et peut être même a-t-il eu tôt connaissance de la traduction ano-

46. Pour Sulpice Sévère, il y a dans l'existence même de Marmoutier une « leçon que Martin a donnée, en tant qu'évêque, à toute la fraction " mondanisée " du clergé et de l'épiscopat gallo-romain " (Vita II, p. 690), et il tend à mettre la description du monastère martinien au service de son propos. 
nyme de la Vita Antonii faite au plus tard en 357 à la demande de moines occidentaux de Trèves ou de Rome ${ }^{47}$. Néanmoins, le "parallèle " qu'établit Sulpice Sévère entre Martin et les moines d'Égypte procède plus d'une volonté d'exalter son héros au-dessus des plus grands ascètes de la vallée du Nil, en réponse d'ailleurs à une contestation de la Vita qui se fait jour dans les milieux gaulois ${ }^{48}$, que d'un souci d'exactitude.

Si l'influence du modèle Égyptien est indéniable en ce qui concerne le monachisme martinien, elle doit être relativisée car deux autres sources ont eu leur importance dans le développement de la pensée anachorétique et cénobitique de Martin. D'une part, lors de son passage à Milan vers 359, il a sans aucun doute eu connaissance de la communauté cléricale d'Eusèbe de Verceil ${ }^{49}$; mais il y eut aussi, et peut être surtout d'autre part, l'influence d'Hilaire de Poitiers.

Lorsque Martin quitte Poitiers à l'issue de son premier séjour auprès d'Hilaire (356), il nous est dit que " c'est dans la tristesse, à ce que l'on rapporte, qu'il entreprit ce lointain voyage, après avoir assuré à ses frères qu'il y subirait bien des épreuves ${ }^{50}$ ". L'usage du mot fratribus permet de comprendre qu'Hilaire vivait entouré d'une communauté - pour laquelle nous n'oserons pas le mot monastique - à laquelle il avait agrégé Martin, et cette expérience de vie communautaire autour de l'évêque ne fut sans doute pas pour peu dans la fondation du "monastère épiscopal » de Marmoutier. Par ailleurs, si l'on ne retrouve pas a priori les hautes spéculations théologiques hilairiennes dans la pastorale martinienne ${ }^{51}$, vue il est vrai uniquement du point de vue de Sulpice Sévère, on doit cependant suivre Jacques Fontaine lorsqu'il suggère que la miséricorde de Martin envers les anciens pécheurs fraternellement accueillis à Marmoutier ${ }^{52}$ reflétait exactement la position

47. DesPrEZ, Vincent, Le Monachisme primitif, op. cit., p. 512.

48. Ce souci de l'hagiographe apparaît déjà dans la Première lettre à Eusèbe (cf. SaintMartin. Textes de Sulpice Sévère, éd. Fontaine, Jacques, Monceaux, Paul, et PIETRI, Luce, Ligugé, 1975, p. 38-41), mais surtout dans le Dialogue I où le récit que Postumianus fait de la vie des moines d'Orient est surtout destiné à montrer la supériorité de Martin (cf. ibid., p. 52-55).

49. Eusèbe évêque de Verceil (†371) entre son ordination épiscopale (345) et son exil en Orient (355) mena une vie régulière commune avec ses clercs et, selon Ambroise de Milan, " le premier en Occident réunit ces éléments divers : la continence du monastère et la discipline de l'Église " (Ep. 63, 66; PL 16, 1207A); sur Eusèbe de Verceil, cf. DE VogüÉ, Adalbert, Histoire littéraire du mouvement monastique dans l'Antiquité, II, Première partie : le monachisme latin. De l'itinéraire d'Égérie à l'éloge funèbre de Népotien (384-396), (Patrimoines christianisme), Paris, 1993, p. 336-338.

50. "Maestus, ut ferunt, peregrinationem illam ingressus est, contestatus fratribus multa aduersa passurum " (Vita, I, p. 262).

51. Desprez, Vincent, "Saint Hilaire de Poitiers, sa vie, son œuvre, ce que Martin doit à Hilaire ", Mémoires de la Société Archéologique de Touraine, LXII, 1997, p. 21-39, sp. p. $37-38$.

52. Pour tenir tête au diable, Martin avait répondu fermement que les fautes anciennes étaient effacées par une meilleure conduite et que, par la miséricorde du Seigneur, il fallait absoudre les péchés de ceux qui avaient cessé de pécher. ("Martinum diabolo repu- 
d'Hilaire telle qu'il l'exprime dans son commentaire des Psaumes ${ }^{53}$ ou dans celui de l'Évangile de saint Matthieu ${ }^{54}$. N'oublions pas non plus la manière dont Hilaire envisage la formation d'un futur évêque :

" Il veut, pour éviter que la faveur trop tôt obtenue ne le rende insolent, que l'épiscope soit d'abord éprouvé par une longue guerre dans les souffrances, il veut qu'il soit promu à ce rang en payant le lourd salaire de l'humilité et de la foi ${ }^{55}$."

Ce qui s'applique assez exactement au moine-évêque Martin.

Enfin, ainsi que l'avance Philippe Henne ${ }^{56}$, la clef pour interpréter le soutien apporté par Hilaire à Martin, c'est la conversion des campagnes restées païennes en ce $\mathrm{IV}^{\mathrm{e}}$ siècle où les villes seules devenaient chrétiennes : en implantant des communautés "monastiques " hors des cités, l'évêque toucherait son peuple là où il vivait.

Différant des modèles antérieurs de monachisme laïc illustrés par Antoine ou Hilarion, Martin, à la fois clerc et moine, représente un nouveau type de vie monastique et l'on peut dire, avec Adalbert de Vogüé, que « ce couple du monachisme et de la cléricature remplace en Occident celui du cénobitisme et de l'érémitisme, qui structurait le milieu monastique égyptien $^{57}$ ". En ce sens, il est un novateur dont les conceptions feront école dans la chrétienté latine.

gnantem respondisse constanter antiqua delicta melioris uitae conuersatione purgari, et per misericordiam Domini absoluendos esse peccatis qui peccare desierint ", Vita, I, p. 300-302).

53. Ps. 137, 3, « [...] peccati confessio professio est desinendi »; HILAIRE DE POITIERS, Tractatus super psalmos, (CSEL, 22), éd. ZINGERLE, A., Vienne, 1891, p. 735.

54. Mt 18, 10, "Ergo uenia omnis ex eo est quo etiam ea quae in se sint peccata post reditum confessionis indulgeat "; HILAIRE DE POITIERS, Sur Matthieu, (Sources chrétiennes, 254 et 258), éd. Dolgnon, Jean, Paris, 1978-1979, II, p. 84.

55. "Id est, ne per recens adeptam regenerationis gratiam insolescat, uult illum multo passionum bello ante temptari, uult multis humilitatis et fidei stipendiis prouehi "; HILAIRE DE PoITIERS, Tractatus super Psalmos. In Psalmum CXVIII, (Corpus Christianorum, Series Latina LXI-A), éd. Doignon, Jean, et Demeulenaere, Roland, Turnhout, 2002, Gimel (III), 18-10, p. 35.

56. HEnne, Philippe, Introduction à Hilaire de Poitiers, suivie d'une anthologie, (Initiation aux Pères de l'Église), Paris, 2006, p. 137.

57. DE VoGüÉ, Adalbert, Histoire littéraire du mouvement monastique dans l'Antiquité, IV, Première partie : le monachisme latin. Sulpice Sévère et Paulin de Nole (393-409), Jérôme, homéliste et traducteur des "Pachomiana ", (Patrimoines christianisme), Paris, 1997, p. 152. 